\title{
ARTICLE OPEN \\ Computational design of bimetallic core-shell nanoparticles for hot-carrier photocatalysis
}

\author{
Luigi Ranno ${ }^{1}$, Stefano Dal Forno $\mathbb{D}^{2}$ and Johannes Lischner ${ }^{3}$
}

Computational design can accelerate the discovery of new materials with tailored properties, but applying this approach to plasmonic nanoparticles with diameters larger than a few nanometers is challenging as atomistic first-principles calculations are not feasible for such systems. In this paper, we employ a recently developed material-specific approach that combines effective mass theory for electrons with a quasistatic description of the localized surface plasmon to identify promising bimetallic core-shell nanoparticles for hot-electron photocatalysis. Specifically, we calculate hot-carrier generation rates of 100 different core-shell nanoparticles and find that systems with an alkali-metal core and a transition-metal shell exhibit high figures of merit for water splitting and are stable in aqueous environments. Our analysis reveals that the high efficiency of these systems is related to their electronic structure, which features a two-dimensional electron gas in the shell. Our calculations further demonstrate that hotcarrier properties are highly tunable and depend sensitively on core and shell sizes. The design rules resulting from our work can guide experimental progress towards improved solar energy conversion devices.

npj Computational Materials (2018)4:31; doi:10.1038/s41524-018-0088-5

\section{INTRODUCTION}

Nanoplasmonic hot-carrier devices are promising for applications in photocatalysis, ${ }^{1-7}$ photovoltaics, ${ }^{8-12}$ and optoelectronics. ${ }^{13-17}$ In these systems, localized surface plasmons are responsible for high sunlight absorption efficiencies. ${ }^{13,18,19}$ The non-radiative decay of such plasmon excitations generates energetic or "hot" electrons and holes, which can be harnessed to trigger otherwise challenging chemical reactions or collected as electric current. ${ }^{20}$

Many nanoplasmonic devices that have been studied are based on metallic nanoparticles, ${ }^{21-25}$ but energy conversion efficiencies have remained relatively low, usually $<1 \%,{ }^{26-30}$ as extraction of hot carriers without significant thermalization losses is challenging. ${ }^{2,31,32}$ It is, therefore, desirable to explore novel device designs. Bimetallic core-shell nanoparticles are natural candidates for improved devices. For instance, Negishi et al. ${ }^{33}$ reported that loading $\mathrm{TiO}_{2}$ with $\mathrm{Au} @ \mathrm{~Pb}$ nanoparticles (i.e., nanoparticles consisting of a gold core and a lead shell) greatly enhances water oxidation activity, while Dai et al. ${ }^{34}$ found that gold nanorods coated with silver exhibit improved surface-enhanced Raman scattering. Core-shell systems exhibit highly tunable optical properties, $^{35}$ but not much is known about their hot carriers. Another challenge is identifying promising candidate systems as the number of possible core-shell nanoparticles that can be obtained by combining different metals and varying core and shell thicknesses is extremely large.

Theory and simulation approaches can accelerate the discovery of novel materials via high-throughput calculations and shed light on microscopic mechanisms that limit achievable energy conversion efficiencies. However, modeling nanoparticles of experimentally relevant sizes is challenging as ab initio approaches, such as density-functional theory, are typically limited to systems containing $<1000$ atoms $^{36}$ (corresponding to a spherical nanoparticle with a radius of only $1 \mathrm{~nm}$ ). To overcome this difficulty, continuum electronic structure methods, such as jellium or effective mass theory, have been used. For example, Manjavacas et al. ${ }^{37}$ developed an approach based on Fermi's golden rule to study plasmon-induced hot carriers in experimentally relevant silver nanoparticles. Recently, Dal Forno, Ranno, and Lischner ${ }^{38}$ extended this approach to other materials and also included an accurate description of hot-carrier lifetimes.

In this paper, we calculate hot-carrier properties of bimetallic core-shell nanoparticles to identify promising candidate systems for photocatalytic applications. In particular, we focus on systems for water splitting, but we expect that our results are also relevant to other reactions, such as $\mathrm{CO}_{2}$ reduction. Searching through the large design space of 100 material combinations with different core and shell thicknesses, we identify design rules for improved devices and discuss how the electronic structure of these systems and their optical properties influence device performance.

\section{RESULTS}

Material screening

To identify bimetallic core-shell nanoparticles with attractive photocatalytic properties, we chose ten metals ( $\mathrm{Ag}, \mathrm{Al}, \mathrm{Au}, \mathrm{Cu}, \mathrm{K}$, $\mathrm{Li}, \mathrm{Na}, \mathrm{Sn}$, and $\mathrm{Zn}$ ) and computed the number of hot electrons and holes that can trigger the hydrogen and oxygen evolution reactions for all possible core-shell combinations. The choice of materials was guided by the following considerations: (i) chemical diversity (the set contains both transition metals and "simple" metals without d-electrons), (ii) experimental relevance and (iii) availability of bulk data for parametrization of our model. Calculations were carried out for nanoparticles with a core radius of $4 \mathrm{~nm}$ and a shell thickness of $1 \mathrm{~nm}$ (chosen to ensure a volume

\footnotetext{
${ }^{1}$ Department of Materials, Imperial College London, London SW7 2AZ, UK; ${ }^{2}$ Department of Physics, Imperial College London, London SW7 2AZ, UK and ${ }^{3}$ Department of Physics and Department of Materials, and the Thomas Young Centre for Theory and Simulation of Materials, Imperial College London, London SW7 2AZ, UK Correspondence: Johannes Lischner (j.lischner@imperial.ac.uk)
}

Received: 24 February 2018 Revised: 14 June 2018 Accepted: 18 June 2018

Published online: 06 July 2018 
(a)

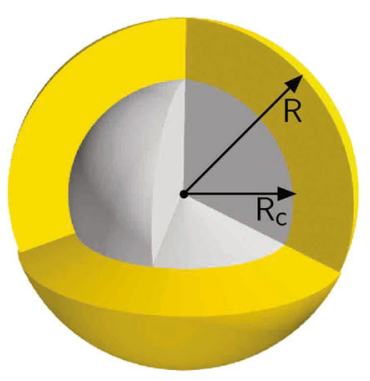

(c)

$$
\text { Hot Holes }
$$

Shell

Li Na K Mg Cu Ag Au Zn Al Sn

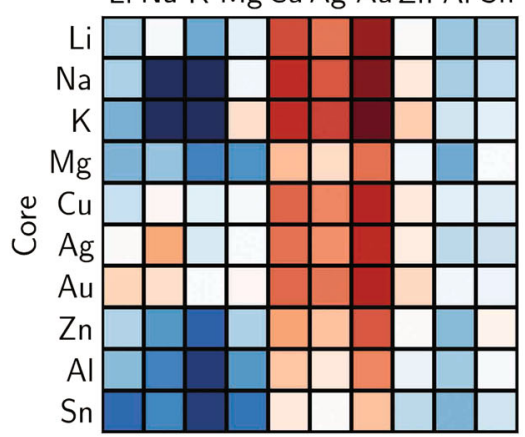

(b)

Hot Electrons

Shell

Li Na K MgCu Ag Au Zn Al Sn

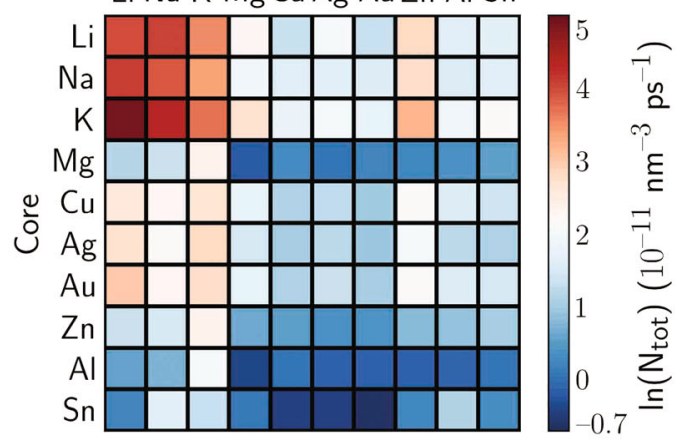

(d)

FoM

Shell

Li Na K MgCuAg AuZn Al Sn

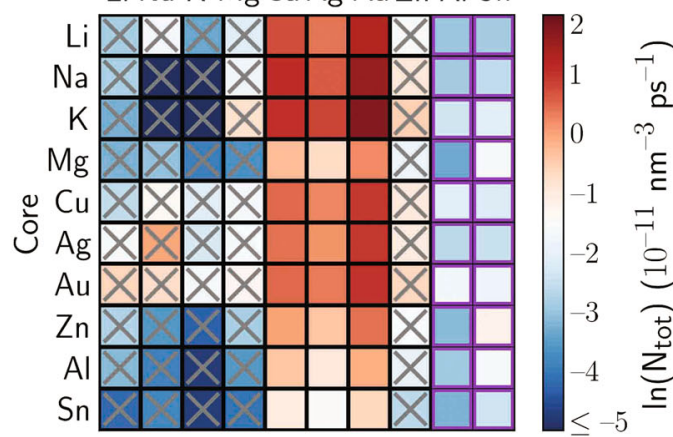

Fig. 1 a Schematic picture of a core-shell nanoparticle (denoted as Ag@Au for a nanoparticle with a Ag core and a Au shell, for example). $R_{c}$ denotes the core radius, while $R$ is the total radius of the particle. b Number of hot electrons above the HER threshold generated per unit time and volume for 100 different core-shell nanoparticles. The row index indicates the core metal and the column index the shell metal. c Same as $\mathbf{b}$, but for hot holes produced below the OER threshold. Note that $\mathbf{c}$, $\mathbf{d}$ have the same colorbar. $\mathbf{d}$ The Figure of Merit of the water splitting reaction, defined as the smaller of the hot-hole and hot-electron numbers in $\mathbf{b}$, $\mathbf{c}$, for the 100 core-shell nanoparticles under consideration. Nanoparticles whose shell oxidizes in an aqueous environment under standard conditions are marked with a purple frame. Nanoparticles whose shell dissolves in an aqueous environment are marked with a gray cross

ratio of core and shell of approximately unity) in an aqueous environment illuminated by sunlight. ${ }^{39}$

Figure 1 shows our results and demonstrates that core-shell nanoparticles often exhibit an improved performance compared to their mono-metallic counterparts (diagonal entries). Figure $1 \mathrm{~b}$ shows the number of hot electrons generated per unit time and volume that can trigger the hydrogen evolution reaction (HER). We find that core-shell nanoparticles containing alkali or alkalineearth metals, such as $\mathrm{K}, \mathrm{Li}, \mathrm{Na}$, and $\mathrm{Mg}$, generate more hot electrons than systems consisting of transition metals. In contrast, core-shell systems containing transition metals are generally better at producing hot holes for the oxygen evolution reaction $(\mathrm{OER})$, see Fig. 1c. Remarkably, the highest generation rates are achieved by core-shell sytems that contain both transition-metal and alkali-metal components. Specifically, core-shell nanoparticles consisting of one $\mathrm{Cu}, \mathrm{Ag}$, or $\mathrm{Au}$ component and one $\mathrm{Li}, \mathrm{Na}$, or $\mathrm{K}$ component perform well.

To split water into oxygen and hydrogen, a photocatalyst has to provide both energetic electrons and holes. To assess the ability of core-shell nanoparticles to trigger this reaction, Fig. 1d shows the Figure of Merit (FoM) defined as the number of hot carrierselectrons or holes-that are produced in the smallest amount by a particular core-shell nanoparticle according to Fig. 1b, c and, therefore, constitute the bottleneck in the water splitting reaction. Again, we find that core-shell nanoparticles, which combine transition-metal and alkali-metal components exhibit the best performance with K@Au and $\mathrm{Na@Au} \mathrm{nanoparticles} \mathrm{having} \mathrm{the}$ highest FoM among all systems that were studied. Core-shell systems consisting of combinations of the transition metals $\mathrm{Cu}$, $\mathrm{Zn}, \mathrm{Ag}$, and $\mathrm{Au}$ also perform quite well, while nanoparticles consisting only of simple-metal components ( $\mathrm{Li}, \mathrm{Na}, \mathrm{K}, \mathrm{Mg}, \mathrm{Al}, \mathrm{Sn}$ ) are not very efficient.

Stability in an aqueous environment is another important characteristic of a useful photocatalyst. In Fig. 1d, the nanoparticles with shell metals that dissolve in water ${ }^{40,41}$ are denoted by a gray cross. Nanoparticles whose shells form an oxide or hydroxide surface layer in water ${ }^{40,41}$ have a purple frame. We find that the core-shell systems with the highest FoM for water splitting are stable in aqueous environments.

Dependence of hot-carrier properties on size of core and shell Having identified promising material combinations for photocatalytic core-shell nanoparticles, we study the dependence of hot-carrier generation rates on the core radius $R_{c}$ and the total radius $R$. Figure 2 shows results for K@Au nanoparticles and demonstrates that increasing $R_{c}$ at fixed $R$ significantly enhances hot-carrier generation rates. The dashed black line in Fig. 2 shows the generation rate for a fixed shell thickness of $0.5 \mathrm{~nm}$. We find that very small nanoparticles $(R<4 \mathrm{~nm})$ generate fewer hot carriers per unit volume than larger systems. At $R=5 \mathrm{~nm}$, the hot-carrier generation rate reaches a maximum and further increase in $R$ leads to a slow decay of the hot-carrier rates. We have found that the optical and electronic properties of other core-shell nanoparticles with both transition-metal and alkali/ 
alkaline-earth components are very similar to the specific case of $\mathrm{K} @ \mathrm{Au}$. Minor differences arise from variations in the material parameters.

Figure 3 shows the energy-resolved spatial distribution of hot carriers for (a) a K@Au nanoparticle and (b) an Au@Na nanoparticle illuminated by monochromatic light at their respective plasmon resonance frequencies. To facilitate extraction of hot carriers and reduce thermalization losses, it is advantageous to generate hot carriers near the surface of the nanoparticle. For the K@Au nanoparticle, we find that indeed most carriers are generated in the Au shell. In contrast, for the Au@Na system a significant fraction of hot carriers, high-energy holes in particular, are generated in the core of the nanoparticle. Interestingly, the hotelectron distribution for this system exhibits a peak at $\sim 1 \mathrm{eV}$

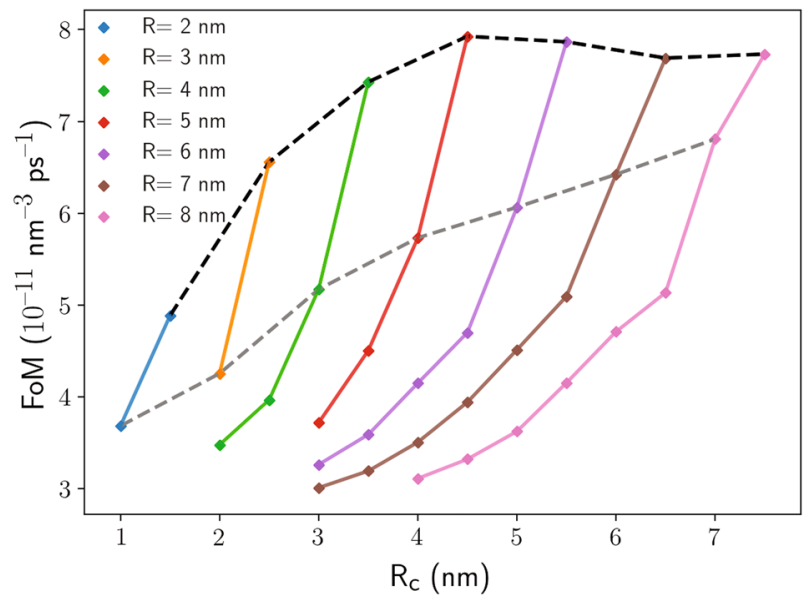

Fig. 2 Dependence of the water splitting Figure of Merit on the core radius $R_{c}$ and the total radius $R$ for a K@Au core-shell nanoparticle The dashed black (gray) line shows the behavior for a fixed shell thickness of $0.5 \mathrm{~nm}(1.0 \mathrm{~nm})$
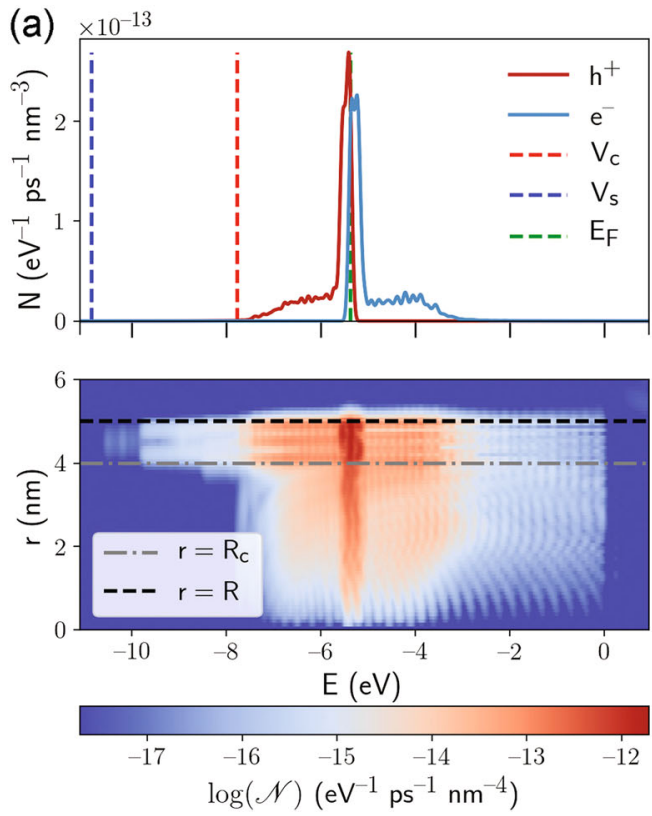

corresponding to the direct injection of hot carriers into states above the vacuum level.

\section{DISCUSSION}

For mono-metallic nanoparticles, we have previously established that the metal work function is a key quantity that characterizes photocatalytic activity. ${ }^{38}$ If the work function is higher than the HER level, all hot electrons can trigger the HER. If, on the other hand, the work function is lower than the OER level, all hot holes can trigger the OER. Core-shell nanoparticles are characterized by two work functions: one for the core metal and one for the shell metal. If these work functions straddle the OER and HER levels, photoexcited electron-hole pairs can trigger both the OER and HER and thus split water. This shows that there is an interesting analogy between bimetallic core-shell nanoparticles and semiconductor photocatalysts, ${ }^{42}$ which can split water if their conduction and valence band edges straddle the OER and HER levels.

Figure 4 shows the work functions of the various core-shell systems that were studied. While alkali and alkaline-earth metals have higher work functions than the HER level, no metal has a lower work function than the OER. Among all metals studied, Au exhibits the lowest work functions (only $\sim 0.2 \mathrm{eV}$ higher than the OER level) indicating that many photoexcited hot holes can still trigger the OER. There is a strong correlation between the coreshell nanoparticles that exhibit a high FoM for water splitting, see Fig. 1d, and the combinations of metals whose work functions (almost) straddle the OER and HER levels. In particular, the simple argument based on work functions correctly predicts that combinations of the transition metals $\mathrm{Au}, \mathrm{Ag}$, and $\mathrm{Cu}$ and the simple metals $\mathrm{K}, \mathrm{Li}$, and $\mathrm{Na}$ yield core-shell nanoparticles with high FoMs for water splitting.

For a given combination of metal components, core-shell nanoparticles can be further categorized into two classes with fundamentally different electronic properties. If the work function of the core metal is larger than the work function of the shell metal, the energy levels of the nanoparticle look similar to the levels of the Au@K system, shown in Fig. 5a. For this system, the
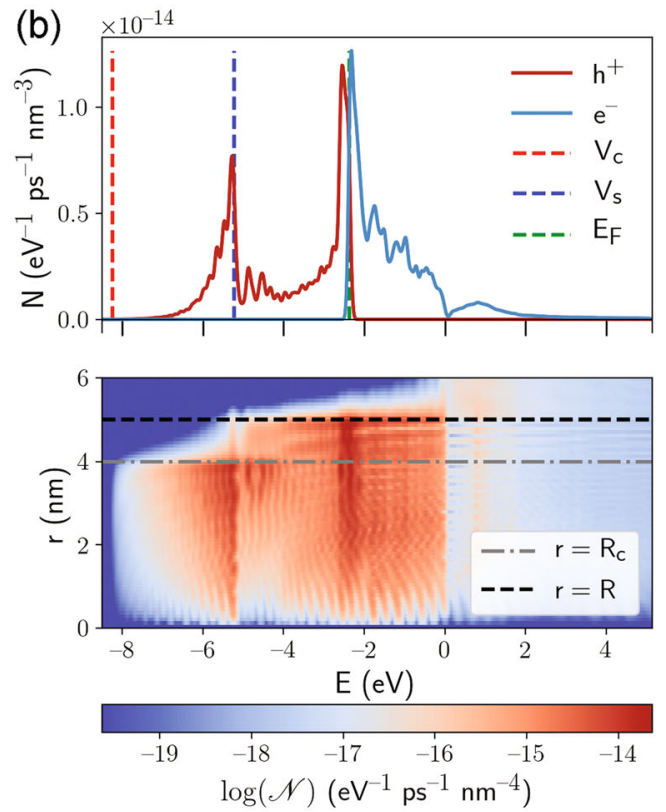

Fig. 3 Energy-resolved spatial distribution $\mathcal{N}$ of hot carriers in a a K@Au nanoparticle with $R_{c}=4 \mathrm{~nm}$ and $R=5 \mathrm{~nm}$ illuminated by monochromatic light with $\hbar \omega=2.25 \mathrm{eV}$ and $\mathbf{b}$ a Au@Na nanoparticle with $R_{\mathrm{c}}=4 \mathrm{~nm}$ and $R=5 \mathrm{~nm}$ illuminated by monochromatic light with $\hbar \omega=3.6 \mathrm{eV}$. The energies of the core $\left(V_{\mathrm{c}}\right)$ and shell $\left(V_{\mathrm{s}}\right)$ potentials as well as the Fermi level $\left(E_{\mathrm{F}}\right)$ are denoted by dashed lines 


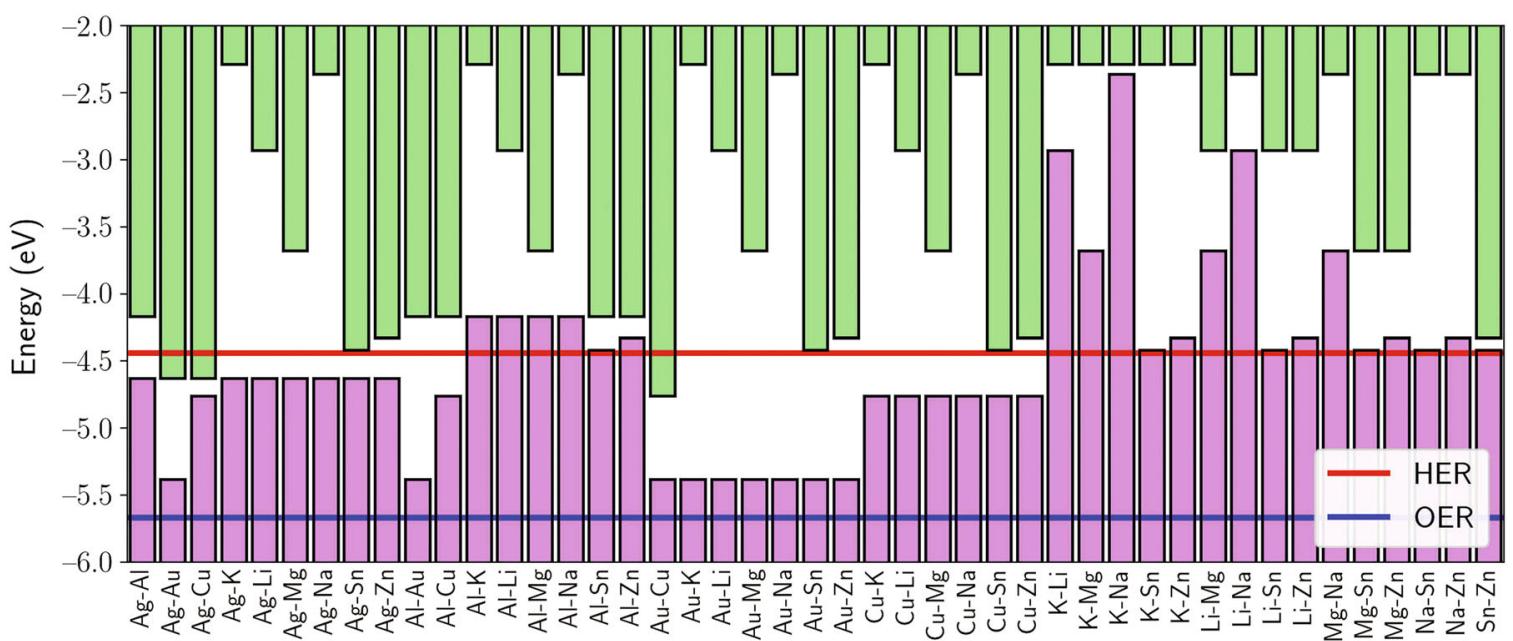

Fig. 4 Work functions of various metal pairs in relation to HER and OER levels. A bimetallic core-shell nanoparticle efficiently splits water when its work functions straddle the OER and HER levels

(a)

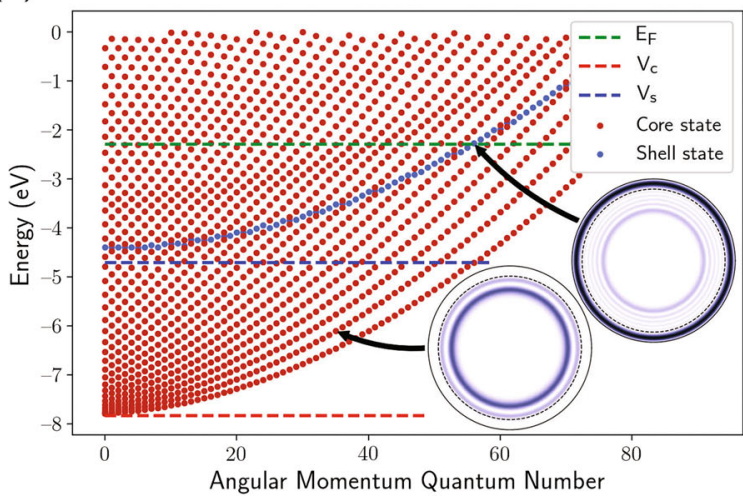

(c)

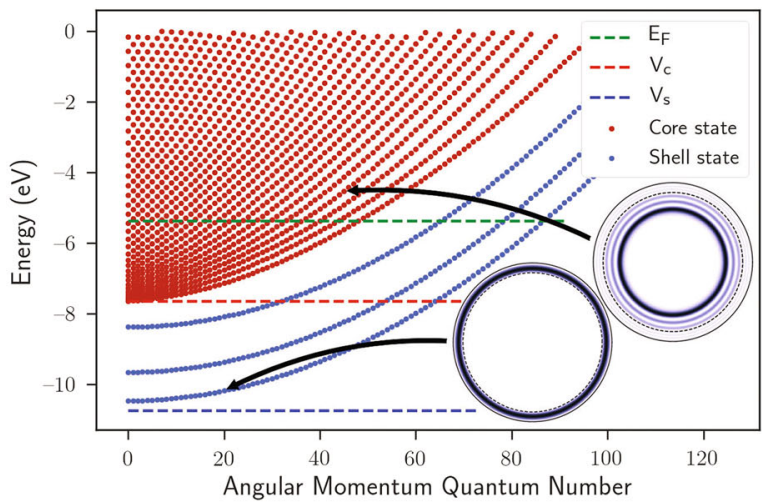

(b)

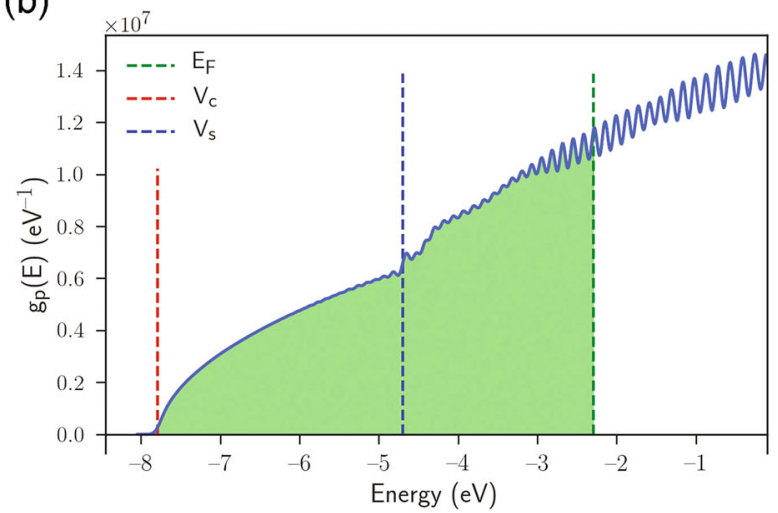

(d)

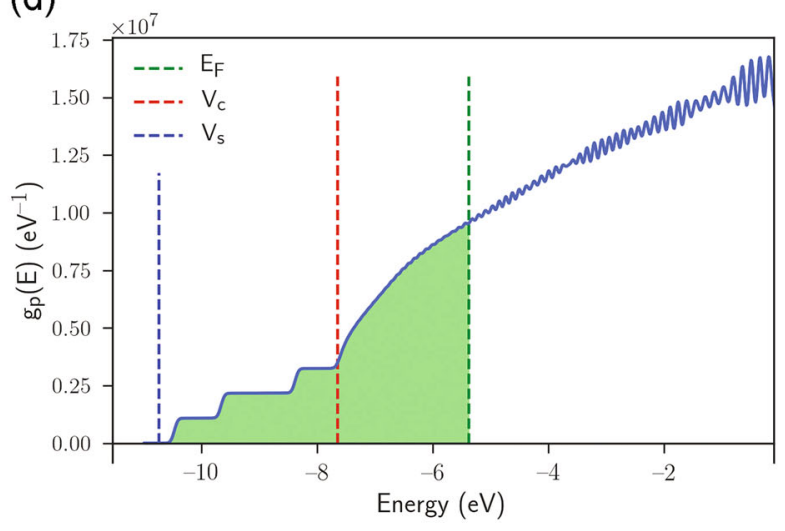

Fig. 5 a Energy level spectrum as function of the angular quantum numberlof a Au@K nanoparticle with core radius of $7 \mathrm{~nm}$ and shell thickness of $1 \mathrm{~nm}$. The depths of the core $\left(V_{c}\right)$ and shell $\left(V_{s}\right)$ potentials and the Fermi level $\left(E_{\mathrm{F}}\right)$ are denoted by dashed lines. Energies of states localized in the core (shell) are denoted by red (blue) dots. Graphs of representative wave functions localized in the core and the shell are shown as insets. b Corresponding density of states (DOS) of a Au@K nanoparticle. The green area denotes states below $E_{\mathrm{F}}$. $\mathbf{c}$, d: same as a, $\mathbf{b}$, but for a K@Au nanoparticle with core radius of $7 \mathrm{~nm}$ and shell thickness of $1 \mathrm{~nm}$

most deeply bound states are localized in the core and the resulting density of states, Fig. $5 b$, at the corresponding energies exhibits a square-root-of-energy behavior, similar to monometallic nanoparticles. ${ }^{38}$ At energies larger than the bottom of the shell potential $V_{s}$ additional states occur that are localized predominantly in the shell of the nanoparticle. Such nanoparticles tend to produce hot carriers localized in the core which are typically more difficult to extract, see Fig. $3 \mathrm{~b}$.

In contrast, Fig. $5 c$ shows the energy levels of a nanoparticle with a shell whose work function is larger than the one of the core. For this system, the deeply bound states are localized in the shell and give rise to a series of steps in the density of states, Fig. $5 d$, 
indicating that the electrons confined in the shell behave similarly to a two-dimensional electron gas. These states form separate branches, which are almost flat near $I=0$. Increasing the value of the angular quantum number / leads to additional nodes in the angular part of the wavefunction. As the wavefunction is localized in the shell, this costs almost no energy. In contrast, increasing the principal quantum number $n$ requires additional nodes in the radial part of the wavefunction. Because of the radial confinement of the states, this is energetically very costly and results in energy gaps between the branches. Such nanoparticles with a deeper shell potential produce predominantly hot carriers in the shell, which are more easily extracted from the device, see Fig. 3a.

Next, we discuss the dependence of the photocatalytic activity on the size of the core and the shell, see Fig. 2. It is well known that the optical properties of core-shell nanoparticles are highly tunable as a function of the volume ratio of core and shell. ${ }^{43,44}$ For example, Fig. 6 shows that a $\mathrm{K}$ core has a significant effect on the absorption cross section of a Au nanoparticle, increasing the total absorbed power (see inset) and consequently the rate of hotcarrier generation. The plasmon energy of a $\mathrm{K}$ nanoparticle is lower than that of a Au nanoparticle and, therefore, the addition of a $\mathrm{K}$ core leads to a redshift of the $\mathrm{Au}$ plasmon resonance improving the overlap with the solar spectrum. This redshift leads to a significant increase in the plasmon amplitude and optical absorption as the imaginary part of the dielectric function of $\mathrm{Au}$ decreases rapidly for energies below $2.5 \mathrm{eV}$. The largest redshift and maximum absorption are achieved for systems with large $\mathrm{K}$ cores and thin Au shells.

Finally, to understand the radius dependence of hot-carrier properties for a fixed shell thickness (see dashed lines in Fig. 2) we

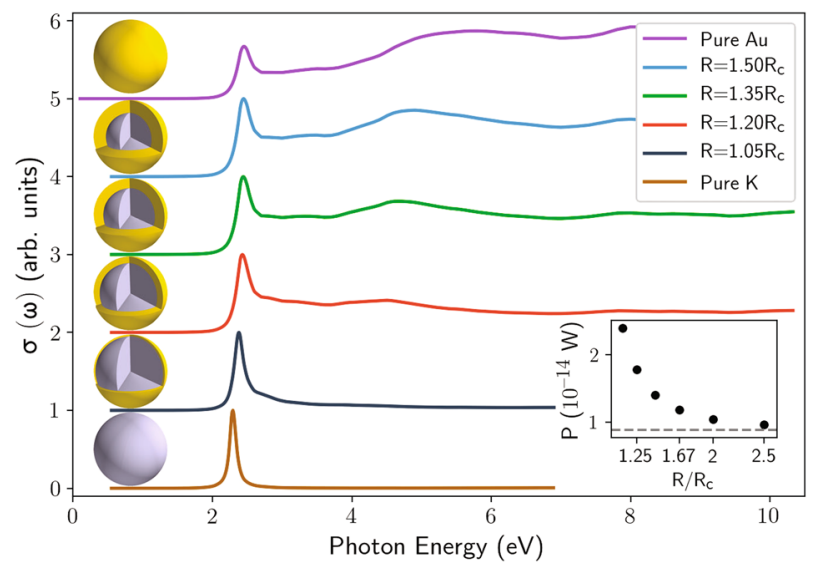

Fig. 6 Absorption cross sections of K@Au core-shell nanoparticles with $R=5 \mathrm{~nm}$, but different core radii $R_{\mathrm{c}}$. Each curve is normalized to a maximum value of unity and offset for clarity. A schematic of the corresponding nanoparticle is shown to the left of each cross section. The inset shows that the total power absorbed by the nanoparticle increases drastically as the shell thickness is reduced. In the inset, the power absorbed by a pure Au nanoparticle is shown as a dashed gray line note that small nanoparticles (with $R$ less than $4 \mathrm{~nm}$ ) have discrete energy levels resulting in fewer but more energetic hot carriers. As the energy level spacing shrinks with increasing $R$, the hot-carrier generation rate also increases. At $R=5 \mathrm{~nm}$, the hot-carrier generation rate reaches a maximum and further increase in $R$ leads to a slow decay as anti-resonant transitions, which result in less useful low-energy carriers, become more dominant. ${ }^{38}$ As the shell thickness increases, the optimal radius also becomes larger since a larger core is required to achieve a significant redshift of the plasmon energy. The interplay of anti-resonant transitions and plasmon redshift as function of core and shell sizes thus determines the optimal radius of the core-shell nanoparticle.

In conclusion, we have studied properties of plasmon-induced hot carriers in bimetallic core-shell nanoparticles for photocatalytic applications. Specifically, we screened 100 different core-shell systems and found that nanoparticles with an alkali-metal core and a transition-metal shell, such as K@Au nanoparticles, exhibit a high Figure of Merit for water splitting and are stable in aqueous environments. Such systems feature hot-hole states that are confined to the shell and behave like a two-dimensional electron gas. In addition, properties of core-shell systems are highly tunable and depend sensitively on the thickness of the shell and the radius of the nanoparticle. Our results, which are summarized in Table 1, thus open up new pathways for designing efficient core-shell nanoparticles for hot-carrier photocatalysis and photovoltaics and provide guidance to experimental efforts towards improved devices.

\section{METHODS}

To study hot carriers in bimetallic core-shell nanoparticles, we extend the approach introduced by Manjavacas et al. ${ }^{37}$ for mono-metallic nanoparticles. In this approach, the total number of hot electrons generated per unit time and volume in a nanoparticle illuminated by sunlight that can trigger the hydrogen evolution reaction is given by

$N_{\text {HER }}=\frac{1}{l_{\text {irr }}} \int_{0}^{+\infty}\left[\int_{E_{\text {HER }}}^{+\infty} N_{e}(E, \omega) d E\right] S(\omega) d \omega$,

where $N_{e}(E, \omega)$ denotes the energy-resolved distribution of hot electrons generated by monochromatic light of angular frequency $\omega$ and intensity $I_{\text {irr. }}$. Also, $S(\omega)$ denotes the solar spectral irradiance ${ }^{39}$ and $E_{\mathrm{HER}}=-4.44 \mathrm{eV}^{45}$ A similar expression provides the total number of holes that can trigger the oxygen evolution reaction. Using Fermi's golden rule, $N_{e}(E, \omega)$ can be expressed as

$$
\begin{aligned}
N_{e}(E, \omega)== & \frac{4 \xi}{\hbar V} \sum_{\mathrm{i}} \sum_{\mathrm{f}}|\langle f|\Phi(\omega)| i\rangle|^{2} \gamma_{\mathrm{if}}\left\{\frac{1}{\left(\hbar \omega-\left[E_{\mathrm{f}}-E_{\mathrm{i}}\right]\right)^{2}+\gamma_{\mathrm{if}}^{2}}\right. \\
& \left.+\frac{1}{\left(\hbar \omega+\left[E_{\mathrm{f}}-E_{\mathrm{i}}\right]\right)^{2}+\gamma_{\mathrm{if}}^{2}}\right\} \delta\left(E-E_{\mathrm{f}}\right),
\end{aligned}
$$

where $i$ and $f$ label initial and final states, respectively, and $V=\frac{4 \pi}{3} R^{3}$ is the volume of the nanoparticle. Also, $E_{\mathrm{i}}$ and $E_{\mathrm{f}}$ denote hole and electron quasiparticle energies, respectively, and $\Phi(\omega)$ is the total electric potential created by the sunlight and the plasmon excitation. Moreover, $\gamma_{\text {if }}$ is the linewidth of the transition and $\xi$ is a parameter chosen to ensure that the power absorbed by the nanoparticle (as calculated from the quasistatic absorption cross section) is equal to the total power of the hot carriers. In

\begin{tabular}{|c|c|c|}
\hline Combination of metals & $\begin{array}{l}\text { Combine transition metals with alkali/alkaline- } \\
\text { earth materials. }\end{array}$ & $\begin{array}{l}\text { Efficient hot-carrier generation when work functions straddle } \\
\text { HER and OER. }\end{array}$ \\
\hline Size of the nanoparticle & Radius between 5 and $10 \mathrm{~nm}$. & $\begin{array}{l}\text { Large enough to avoid quantum size effects, small enough to } \\
\text { avoid anti-resonant transitions. }\end{array}$ \\
\hline
\end{tabular}

Table 1. Rules for designing efficient core-shell nanoparticles for hot-carrier photocatalysis 
our calculations, the $\delta$-function is replaced by a Gaussian with a standard deviation of $0.05 \mathrm{eV}$ and we use $l_{\text {irr }} \approx 1.33 \mathrm{~mW} \mathrm{~m}^{-2}$ in Eq. (1).

To evaluate Eq. (2) for a core-shell nanoparticle, we solve the Schrödinger's equation for a two-step spherical potential well. The depths of the two wells are determined by the conditions that (i) the work function of the core-shell system must equal the work function of the shell material $^{46,47}$ and (ii) the difference between the core and shell depths is equal to the Volta potential. Note that this approach typically describes well electrons in s- and p-bands, but fails to capture d-electrons, which can play an important role in transition metals. ${ }^{38}$ As a consequence, our calculations yield valuable lower bounds on hot-carrier generation rates. Photoexcited d-electrons contribute significantly to hot-carrier rates when the photon energy is larger than the energy difference between the Fermi energy and the highest occupied d-band. ${ }^{48}$ Additional work is needed to develop accurate electronic structure models for experimentally relevant transition-metal nanoparticles. Note that the two-step potential describes a hard interface between the core and the shell. Experimentally, we expect that some interfaces, especially those between highly miscible metals, are smooth. However, the effect of interface smoothness on hot-carrier properties is small. For example, Manjavacas et al. ${ }^{37}$ obtained very similar hot-carrier generation rates for an abrupt potential well and a smoother mean-field potential that includes effects of electron-electron interactions. The plasmon potential $\Phi$ for a core-shell nanoparticle is calculated in the quasistatic approximation using experimental bulk dielectric functions. ${ }^{49,50}$ It is well known that the quasistatic approximation is only valid if (i) the nanoparticle is smaller than the wavelength of light and (ii) the nanoparticle is sufficiently large that quantum size effects can be neglected. Moreover, the quasistatic approximation underestimates the width of the localized surface plasmon resonance in the absorption spectrum, ${ }^{51,52}$ but the effect of this correction on hot-carrier rates is negligible. ${ }^{53}$ The transition linewidths $\gamma_{\text {if }}$ are obtained from the hot-carrier lifetimes and include effects of electron-electron and electron-phonon scattering. ${ }^{38}$ We calculate the lifetimes $\tau_{\mathrm{CS}}$ of hot carriers in core-shell nanoparticles from the lifetimes of the core material $\tau_{C}$ and the shell material $\tau_{\mathrm{S}}$ via

$\frac{1}{\tau_{\mathrm{CS}}}=\frac{\zeta}{\tau_{\mathrm{C}}}+\frac{1-\zeta}{\tau_{\mathrm{S}}}$.

Here, $\zeta$ is given by

$$
\zeta=\frac{V_{\mathrm{C}}\left[g_{\mathrm{C}}\left(E_{\mathrm{F}}\right)^{p}\right.}{V_{\mathrm{C}}\left[g_{\mathrm{C}}\left(E_{\mathrm{F}}\right)\right]^{p}+V_{\mathrm{S}}\left[g_{\mathrm{S}}\left(E_{\mathrm{F}}\right)\right]^{p}},
$$

where $g_{\mathrm{C}}\left(E_{\mathrm{F}}\right)\left(g_{\mathrm{S}}\left(E_{\mathrm{F}}\right)\right)$ denotes the density of states at the Fermi level of the core (shell) material and the exponent $p$ is set to 1 for electron-phonon scattering and to -2.5 for electron-electron scattering. ${ }^{54,55}$ Further methodological details and all material-specific parameters that were used in our calculations are available in the Supplementary Information.

\section{Data availability}

The data and code used in this study are available from the corresponding author upon request.

\section{ACKNOWLEDGEMENTS}

S.D.F. and J.L. acknowledge support from EPSRC under Grant No. EP/N005244/1 and also from the Thomas Young Center under Grant No.TYC-101. Via J.L.'s membership of the UK's HEC Materials Chemistry Consortium, which is funded by EPSRC (EP/ L000202), this work used the ARCHER UK National Supercomputing Service. S.D.F. and J.L. acknowledge support from EPSRC under Grant No. EP/N005244/1 and also from the Thomas Young Center under Grant No.TYC-101.

\section{AUTHOR CONTRIBUTIONS}

L. R. performed calculations of hot-carrier rates for core-shell nanoparticles. L. R. and S. D. F. developed the computer code for these calculations. L. R., S. D. F., and J. L. contributed to the interpretation of the results and the writing of the manuscript.

\section{ADDITIONAL INFORMATION}

Supplementary information accompanies the paper on the npj Computational Materials website (https://doi.org/10.1038/s41524-018-0088-5).

Competing interests: The authors declare no competing interests.
Publisher's note: Springer Nature remains neutral with regard to jurisdictional claims in published maps and institutional affiliations.

\section{REFERENCES}

1. Ahn, W., Ratchford, D. C., Pehrsson, P. E. \& Simpkins, B. S. Surface plasmon polariton-induced hot carrier generation for photocatalysis. Nanoscale 9, 3010-3022 (2017)

2. Anjaneyulu, O. et al. Plasmon-mediated photothermal conversion by TiN nanocubes toward $\mathrm{CO}$ oxidation under solar light illumination. RSC Adv. 6, 110566-110570 (2016).

3. Chen, Y. -C. et al. Au@Nb@ $\mathrm{H}_{x} \mathrm{~K}_{1-x} \mathrm{NbO}_{3}$ nanopeapods with near-infrared active plasmonic hot-electron injection for water splitting. Nat. Commun. 9, 232 (2018).

4. Hou, B., Shen, L., Shi, H., Kapadia, R. \& Cronin, S. B. Hot electron-driven photocatalytic water splitting. Phys. Chem. Chem. Phys. 19, 2877-2881 (2017).

5. Ueno, K., Oshikiri, T., Murakoshi, K., Inoue, H. \& Misawa, H. Plasmon-enhanced light energy conversion using gold nanostructured oxide semiconductor photoelectrodes. Pure Appl. Chem. 87, 547-555 (2015).

6. Ueno, K., Oshikiri, T. \& Misawa, H. Plasmon-induced water splitting using metallicnanoparticle-loaded photocatalysts and photoelectrodes. Chemphyschem 17, 199-215 (2016).

7. Zhang, Q. et al. Recent advancements in plasmon-enhanced visible light-driven water splitting. J. Mater. 3, 33-50 (2017).

8. García de Arquer, F. P., Mihi, A., Kufer, D. \& Konstantatos, G. Photoelectric energy conversion of plasmon-generated hot carriers in metal-insulator-semiconductor structures. ACS Nano 7, 3581-3588 (2013).

9. Gong, T., Krayer, L. \& Munday, J. N. Design concepts for hot carrier-based detectors and energy converters in the near ultraviolet and infrared. J. Photonics Energy 6, 042510 (2016).

10. Lee, Y. K. et al. Surface plasmon-driven hot electron flow probed with metalsemiconductor nanodiodes. Nano Lett. 11, 4251-4255 (2011).

11. Robatjazi, H., Bahauddin, S. M., Doiron, C. \& Thomann, I. Direct plasmon-driven photoelectrocatalysis. Nano Lett. 15, 6155-6161 (2015).

12. Shokri Kojori, H. et al. Plasmon field effect transistor for plasmon to electric conversion and amplification. Nano Lett. 16, 250-254 (2016).

13. Ai, Y., Nguyen, V. Q., Ghilane, J., Lacaze, P. C. \& Lacroix, J. C. Plasmon-induced conductance switching of an electroactive conjugated polymer nanojunction. ACS Appl. Mater. Interfaces 9, 27817-27824 (2017).

14. Brongersma, M. L. Plasmonic photodetectors, photovoltaics, and hot-electron devices. Proc. IEEE 104, 2349-2361 (2016).

15. Gong, T. \& Munday, J. N. Aluminum-based hot carrier plasmonics. Appl. Phys. Lett. 110, 021117 (2017).

16. Lu, R. et al. A localized surface plasmon resonance and light confinementenhanced near-infrared light photodetector. Laser Photonics Rev. 602, 595-602 (2016).

17. Sharma, A. et al. Plasmon induced ultrafast injection of hot electrons in $\mathrm{Au}$ nanoislands grown on a CdS film. J. Mater. Chem. C5, 618-626 (2017).

18. Lv, W., Phelan, P. E., Swaminathan, R., Otanicar, T. P. \& Taylor, R. A. Multifunctional core-shell nanoparticle suspensions for efficient absorption. J. Sol. Energy Eng. 135, 021004 (2012).

19. Raji, R., Sibi, K. S. \& Gopchandran, K. G. ZnO:Ag nanorods as efficient photocatalysts: Sunlight driven photocatalytic degradation of sulforhodamine B. Appl. Surf. Sci. 427, 863-875 (2018).

20. Kim, S. M., Lee, W. S., Moon, S. Y. \& Park, J. Y. The effect of hot electrons and surface plasmons on heterogeneous catalysis. J. Phys. Condens. Matter 28, 254002 (2016).

21. Li, H. et al. Surface-plasmon-resonance-enhanced photoelectrochemical water splitting from Au-nanoparticle-decorated $3 \mathrm{D} \mathrm{TiO}_{2}$ nanorod architectures. J. Phys. Chem. C. 121, 12071-12079 (2017).

22. Liu, Z., Hou, W., Pavaskar, P., Aykol, M. \& Cronin, S. B. Plasmon resonant enhancement of photocatalytic water splitting under visible illumination. Nano Lett. 11, 1111-1116 (2011).

23. Maksymov, I. S. \& Greentree, A. D. Plasmonic nanoantenna hydrophones. Sci. Rep. 6, 32892 (2016).

24. Oka, H. Highly-efficient entangled two-photon absorption with the assistance of plasmon nanoantenna. J. Phys. B: At., Mol. Opt. Phys. 48, 115503 (2015).

25. Qi, Z. et al. Au nanoparticle-decorated silicon pyramids for plasmon-enhanced hot electron near-infrared photodetection. Nanotechnology 28, 275202 (2017).

26. Knight, M. W., Sobhani, H., Nordlander, P. \& Halas, N. J. Photodetection with active optical antennas. Science 332, 702-704 (2011).

27. Knight, M. W. et al. Embedding plasmonic nanostructure diodes enhances hot electron emission. Nano. Lett. 13, 1687-1692 (2013).

28. Mubeen, S. et al. An autonomous photosynthetic device in which all charge carriers derive from surface plasmons. Nat. Nanotechnol. 8, 247-251 (2013). 
29. Reineck, P. et al. A solid-state plasmonic solar cell via metal nanoparticle selfassembly. Adv. Mater. 24, 4750-4755 (2012).

30. Valenti, M. et al. Hot carrier generation and extraction of plasmonic alloy nanoparticles. ACS Photonics 4, 1146-1152 (2017).

31. Hartland, G. H. Optical studies of dynamics in noble metal nanostructures. Chem. Rev. 111, 3858-3887 (2011).

32. Wu, K., Chen, J., McBride, J. R. \& Lian, T. Efficient hot-electron transfer by a plasmoninduced interfacial charge-transfer transition. Science 349, 632-635 (2015).

33. Negishi, R., Naya, S. I., Kobayashi, H. \& Tada, H. Gold(Core)-lead(Shell) nanoparticle-loaded titanium(IV) oxide prepared by underpotential photodeposition: plasmonic water oxidation. Angew. Chem. - Int. Ed. 56, 10347-10351 (2017).

34. Dai, L. et al. Bimetallic Au/Ag core-shell superstructures with tunable surface plasmon resonance in the near-infrared region and high performance surfaceenhanced Raman scattering. Langmuir 33, 5378-5384 (2017).

35. Cortie, M. B. \& McDonagh, A. M. Synthesis and optical properties of hybrid and alloy plasmonic nanoparticles. Chem. Rev. 111, 3713-3735 (2011).

36. Reimers, J. Computational Methods for Large Systems: Electronic Structure Approaches for Biotechnology and Nanotechnology, (Hoboken, New Jersey, USA, Wiley, 2011).

37. Manjavacas, A., Liu, J. G., Kulkarni, V. \& Nordlander, P. Plasmon-induced hot carriers in metallic nanoparticles. ACS Nano 8, 7630-7638 (2014).

38. Dal Forno, S., Ranno, L. \& Lischner, J. Material, size and environment dependence of plasmon-induced hot carriers in metallic nanoparticles. J. Phys. Chem. C. 122 8517-8527 (2018).

39. ASTM International. ASTM G173-03(2012), Standard Tables for Reference Solar Spectral Irradiances: Direct Normal and Hemisphericalon $37^{\circ}$ Tilted Surface. Technical report. (ASTM International, West Conshohocken, PA, 2012).

40. Beverskog, B. Revised pourbaix diagrams for copper at $25^{\circ}$ to $300{ }^{\circ} \mathrm{C}$. J. Electrochem. Soc. 144, 3476 (1997).

41. Takeno, N. Atlas of Eh-pH diagrams: Intercomparison of thermodynamic databases (Geological Survey of Japan Open File Report No.419). Technical report. (National Institute of Advanced Industrial Science and Technology Research Center for Deep Geological Environments, Tokyo, Japan, 2005).

42. Stevanović, V., Lany, S., Ginley, D. S., Tumas, W. \& Zunger, A. Assessing capability of semiconductors to split water using ionization potentials and electron affinities only. Phys. Chem. Chem. Phys. 16, 3706-3714 (2014).

43. Lu, L., Burkey, G., Halaciuga, I. \& Goia, D. V. Core-shell gold/silver nanoparticles: Synthesis and optical properties. J. Colloid Interface Sci. 392, 90-95 (2013).

44. Zhu, J. Theoretical study of the optical absorption properties of Au-Ag bimetallic nanospheres. Phys. E: Low.-Dimens. Syst. Nanostruct. 27, 296-301 (2005).
45. Trasatti, S. The absolute electrode potential: an explanatory note (Recommendations 1986). Pure Appl. Chem. 58, 955-966 (1986).

46. Holmberg, N., Laasonen, K. \& Peljo, P. Charge distribution and Fermi level in bimetallic nanoparticles. Phys. Chem. Chem. Phys. 18, 2924-2931 (2016).

47. Peljo, P., Manzanares, J. A. \& Girault, H. H. Contact potentials, fermi level equilibration, and surface charging. Langmuir 32, 5765-5775 (2016).

48. Gong, T. \& Munday, J. N. Materials for hot carrier plasmonics [Invited]. Opt. Mater. Express 5, 2501-2512 (2015).

49. Sihvola, A. \& Lindell, I. V. Transmission line analogy for calculating the effective permittivity of mixtures with spherical multilayer scatterers. J. Electromagn. Waves Appl. 2, 741-756 (1988).

50. Sihvola, A. Character of surface plasmons in layered spherical structures. Progress. Electromagn. Res. 62, 317-331 (2006).

51. Kreibig, U. \& Vollmer, M. Optical Properties of Metal Clusers. (Springer Series in Materials Science 25, Springer, Berlin, 1995).

52. Berciaud, S., Cognet, L., Tamarat, P. \& Lounis, B. Observation of intrinsic size effects in the optical response of individual gold nanoparticles. Nano Lett. 5 515-518 (2005).

53. Molina, R. A., Weinmann, D. \& Jalabert, R. A. Oscillatory size dependence of the surface plasmon linewidth in metallic nanoparticles. Phys. Rev. B 65, 155427 (2002).

54. Hodak, J. H., Henglein, A. \& Hartland, G. V. Tuning the spectral and temporal response in PtAu core-shell nanoparticles. J. Chem. Phys. 114, 2760-2765 (2001)

55. Echenique, P. M., Pitarke, J. M., Chulkov, E. V. \& Rubio, A. Theory of inelastic lifetimes of low-energy electrons in metals. Chem. Phys. 251, 1-35 (2000).

Open Access This article is licensed under a Creative Commons Attribution 4.0 International License, which permits use, sharing, adaptation, distribution and reproduction in any medium or format, as long as you give appropriate credit to the original author(s) and the source, provide a link to the Creative Commons license, and indicate if changes were made. The images or other third party material in this article are included in the article's Creative Commons license, unless indicated otherwise in a credit line to the material. If material is not included in the article's Creative Commons license and your intended use is not permitted by statutory regulation or exceeds the permitted use, you will need to obtain permission directly from the copyright holder. To view a copy of this license, visit http://creativecommons. org/licenses/by/4.0/.

(c) The Author(s) 2018 